\title{
Climate variability and malaria transmission - Fogera district, Ethiopia, 2003-2011
}

\author{
Addisu Workineh Kassa ${ }^{1}$, Belay Bezabih Beyene ${ }^{2}$ \\ ${ }^{1}$ Public health emergency management, Bahir Dar, Ethiopia \\ ${ }^{2}$ Amhara regional health bureau, Bahir Dar, Ethiopia \\ Email address: \\ analid9800@gmail.com (A. W. Kassa), analid9800@yahoo.com (A. W. Kassa)
}

\section{To cite this article:}

Addisu Workineh Kassa, Belay Bezabih Beyene. Climate Variability and Malaria Transmission - Fogera District, Ethiopia, $2003-2011$. Science Journal of Public Health. Vol. 2, No. 3, 2014, pp. 234-237. doi: 10.11648/j.sjph.20140203.26

\begin{abstract}
Background: Epidemic malaria creates serious public health emergencies in Ethiopia. Malaria transmission rates have been linked to temperature and precipitation; factors that will be affected by increasing climate variability. Method: We analyzed climate and malaria data from January 2003-December 2011 in Fogera district of Ethiopia. We compared monthly rainfall and temperature data with the number of confirmed malaria cases. Results: We identified 104,716 confirmed malaria cases, which were treated in the district from 2003-2011 (monthly cases range from 98 - 5038). The annual prevalence rate was 104 per 1000. Annual average rainfall was $1268 \mathrm{~mm}$, and annually there were between four and six months with rainfall that exceeded $80 \mathrm{~mm}$. There was a trend associating months with rainfall above $80 \mathrm{~mm}$ and higher rates of confirmed malaria cases in seven of the nine years of the study period. Average monthly temperatures throughout the nine years period ranged from $18^{\circ} \mathrm{c}$ to $28^{\circ} \mathrm{c}$. Conclusions: Malaria still constitutes a serious public health problem in Fogera district. Monthly precipitation greater than $80 \mathrm{~mm}$ was associated with increased malaria transmission rates in the district, and temperature probably was not a limiting factor. We recommend the development of climate prediction models to help forecast and control malaria outbreaks in the district.
\end{abstract}

Keywords: Malaria, Climate, Fogera

\section{Introduction}

Malaria is endemic in over 100 countries world-wide. Ninety per cent of malaria deaths occur in countries in sub-Saharan Africa. In many countries of sub-Saharan Africa, malaria has stable transmission with high rates of infection and in areas of unstable malaria endemic; transmission tends to be seasonal with short epidemics of varying intensity[1].

Ethiopia is one of the countries in sub-Saharan, where altitude and climatic factors such as, rainfall, and humidity are important determinants of malaria epidemiology[2].

Malaria is a preventable and curable disease. However, in order to be able to prevent or cure the disease in the context of malaria outbreaks in Ethiopia, decision-makers need to be aware of the risk of outbreaks occurring:

- in space (which areas under their jurisdiction are most likely to suffer a malaria outbreak)

- in time (in which months/years are malaria outbreaks most likely to occur)[3]

Malaria outbreaks are some of the most serious public health emergencies with which health officials are confronted[4]. Early identification of malaria outbreak is an important first step towards implementing effective disease interventions and reducing resulting morbidity and mortality[5].

Many factors play a role in the distribution of malaria; climate variability has shown to be a major determinant.

Rainfall is one of climate factors plays important roles in malaria epidemiology because of water not only provide medium for aquatic stage of mosquito life cycle but also increase the relative humidity and then the longevity of adult mosquitoes[6].

Temperature is the second climate factor affects directly the developmental period in the life cycle of mosquitoes; blood feeding rate, gonotrophic cycle and longevity[7].

The hierarchical system proposed for malaria early warning system takes account of all the different ranges of forecasts which can be developed to suit the various needs of the health sector:

- Long range predictions based on seasonal climate forecasts. 
- Short range predictions based on active monitoring of risk factors (e.g. temperature and rainfall)[8], [9].

Early detection of outbreaks using disease monitoring; there is no lead-time. But this approach provides specific information on timing and location of an outbreak[10].

Therefore analysis of the space-time effect of this association has practical and is crucial steps to explore effect of climate variability, and malaria prevalence on local malaria epidemiology.

\section{Method}

Both climate and malaria data between January 2003-December 2011 was used in this study. Climate parameters that were used include; monthly rainfall and temperature. Climate suitability for malaria transmission was defined as the coincidence of precipitation accumulation greater than $80 \mathrm{~mm}$, mean temperature between $18^{\circ} \mathrm{c}$ and $32^{\circ} \mathrm{c}$, and relative humidity between $60-80$ percent. We compared monthly rainfall and temperature data with the number of confirmed malaria cases. Laboratory confirmed malaria cases were summed over both age groups.

\section{Study Area, Population and Period}

Fogera district with population of 206,730 is found at altitude ranges $1820-2040 \mathrm{~ms}$ above sea level. The mean annual rain fall is $1284 \mathrm{~mm}$; and monthly average temperature ranges $18-28^{\circ} \mathrm{c}$. The district malaria surveillance with climate data included from January 2003 to December 2011.

Malaria is diagnosed using direct microscopy in hospitals and health centres and use of rapid diagnostic test in health posts.

The collected data were manually checked for completeness for each variable and subsequently entered into a computer. Then prominent findings of the analysis were presented on a table and figures.

\section{Results}

We identified 104,716 confirmed malaria cases, which were treated in the district from 2003-2011 (monthly cases range from 98-5038) with the annual prevalence rate 104 per 1000 and annual average rainfall was $1268 \mathrm{~mm}$. There was a trend associating months with rainfall above $80 \mathrm{~mm}$ and higher rates of confirmed malaria cases in seven of the nine years of the study period.

The results of this study show that seasonality of climate influences the variation in malaria transmission in the district. It is observed that rainfall plays an important role in the distribution of malaria transmission.

Table 1. Malaria attack rate per 1000 populations of Fogera, Amhara, from 2003-2011.

\begin{tabular}{|c|c|c|c|c|c|c|c|c|c|c|c|c|c|}
\hline \multirow{2}{*}{ Year } & \multicolumn{12}{|c|}{ Attack rate per 1000 population } & \multirow{2}{*}{$\begin{array}{c}\text { Population a } \\
\text { risk }\end{array}$} \\
\hline & Jan & Feb & Mar & Apr & May & June & July & Aug. & Sep & Oct & Nov. & Dec & \\
\hline 2003 & 2.2 & 2.3 & 2.1 & 3.6 & 3.9 & 3.8 & 1.6 & 1.6 & 1.2 & 1.0 & 1.1 & 1.7 & $197,018 *$ \\
\hline 2004 & 1.4 & 0.8 & 1.0 & 1.0 & 1.0 & 1.0 & 4.6 & 1.4 & 2.2 & 4.6 & 5.1 & 6.2 & $221,996^{*}$ \\
\hline 2005 & 4.2 & 4.0 & 4.3 & 4.5 & 5.6 & 5.5 & 8.5 & 8.0 & 9.4 & 10.0 & 13.8 & 9.2 & $241,488 *$ \\
\hline 2006 & 9.6 & 5.1 & 4.4 & 4.7 & 4.7 & 12.0 & 4.5 & 3.4 & 4.2 & 1.2 & 2.5 & 1.8 & $191,219 * *$ \\
\hline 2007 & 2.4 & 2.2 & 1.1 & 2.3 & 2.4 & 2.3 & 0.5 & 0.8 & 0.8 & 0.8 & 0.9 & 0.7 & 194,219 \\
\hline 2008 & 0.9 & 0.9 & 0.8 & 0.9 & 0.8 & 0.5 & 2.0 & 3.2 & 2.0 & 9.8 & 9.5 & 0.7 & 197,269 \\
\hline 2009 & 3.9 & 5.4 & 5.4 & 4.7 & 4.1 & 7.4 & 19.5 & 15.6 & 10.7 & 25.1 & 20.0 & 10.0 & 200,371 \\
\hline 2010 & 5.8 & 3.1 & 3.3 & 3.7 & 6.4 & 7.6 & 1.5 & 1.4 & 3.4 & 5.3 & 6.0 & 5.1 & 203,524 \\
\hline 2011 & 4.0 & 3.9 & 4.1 & 3.9 & 5.0 & 6.1 & 4.2 & 5.6 & 8.8 & 9.6 & 7.6 & 4.5 & 206,730 \\
\hline
\end{tabular}

* Projected population before 2007 census

**Woreta town departed from Fogera district and became a district in 2006

Temperature throughout the nine years ranged from $18^{\circ} \mathrm{c}$ to $28^{0} \mathrm{c}$ except November $2006\left(13.2^{0} \mathrm{c}\right)$ and $4-6$ months in a year average monthly rainfall was between $119.4-503 \mathrm{~mm}$ with the onset month June and cessation month October/November, so temperature was probably not a limiting factor for malaria transmission in the district.

\section{Discussion}

The major malaria transmission was from September to November, following the main rainy season and a shorter transmission seasons from April to May following the short rainy seasons.

Time series analysis on monthly scale from 2003 to 2011 showed high number of malaria cases reported from September to November.

Over the last nine years January 2003 to December 2011, 104,716 confirmed malaria cases treated in the district with annual average 11,635 and monthly 970 confirmed malaria cases reported.

Temperature throughout the nine years ranges from $18^{0} \mathrm{c}$ to $28^{0} \mathrm{c}$ except November $2006\left(13.2^{\circ} \mathrm{c}\right)$ and $4-6$ months in a year average monthly rainfall was between $119.4-503 \mathrm{~mm}$ with the onset month June while the cessation month October/November. The rainfall distribution pattern was going to be one of the major determinants of malaria transmission. 


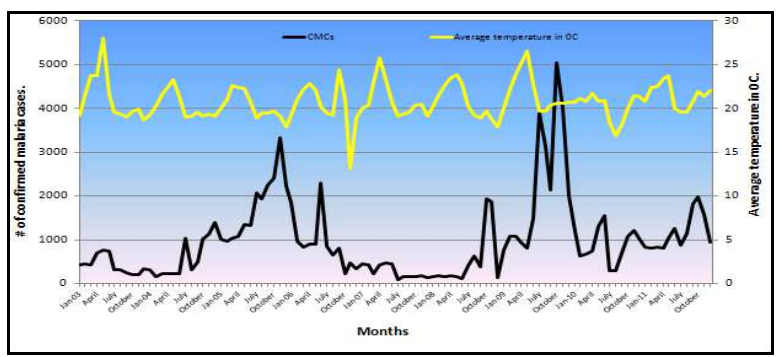

Figure 1. Confirmed malaria cases with average monthly temperature of Fogera, Amhara, Ethiopia, from 2003-2011.

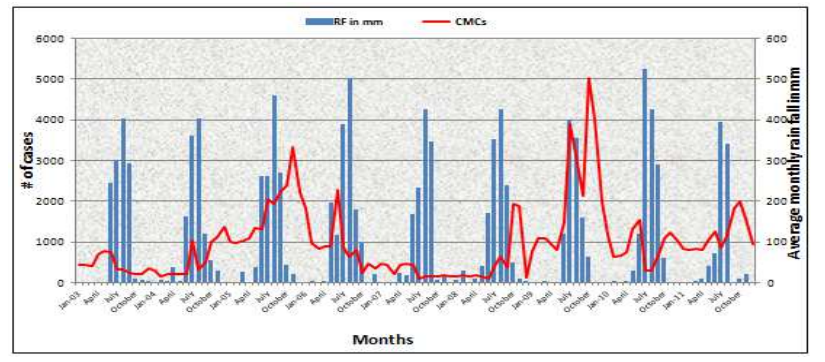

Figure 2. Confirmed malaria cases with average monthly rain fall of Fogera, Amhara, Ethiopia, from 2003-2011.

A steep rise in the number of laboratory confirmed malaria cases occurred in August 2004 to December 2005\& January 2009 to November 2009. Within these years October \& November were the peak months. During these peak seasons average monthly temperature was conducive for increasing mosquito density which was between $18.86-25.73^{\circ}{ }_{\mathrm{C}}$.

The table and figures above show the rainfall, temperature and malaria distribution from January 2003 to December 2011.The spatial patterns indicate that there was a decrement trend from July 2006 to September 2008; this might be due to supply of new long lasting impregnated nets (LLINs) to the community and coartem supply to the health extension workers and managing at community level. But starting from November 2008 to November 2009 shows increments this might be old LLINs not replaced at that time and the resistance of DDT for Anopheles mosquitoes. Due to resistance of malaria vectors to dichlorodiphenyltetrachloro ethane (DDT), the use of this insecticide for indoor residual spray (IRS) has been discontinued in 2009. Deltametrin was used as an interim substitute insecticide for DDT for IRS operations. However, the selection of insecticides for IRS use in Ethiopia will be determined annually based on the insecticide resistance pattern of the vectors and other factors. Additionally, extensive resistance to deltametrin and Malathion was reported. This might be the reason why laboratory confirmed malaria cases increased in number starting from November 2010 besides other factors.

\section{Conclusion}

Climate variability is widely considered to be a major driver of inter-annual variability of malaria incidence in Ethiopia. The relationships of variability in rainfall and temperatures to malaria transmission in Fogera were assessed in this study. Malaria was a serious public health problem in Fogera. It was responsible for 44.5\% (20183) outpatient visit to health facilities (December 2011). In this study, climate suitability for malaria transmission was defined as the coincidence of precipitation accumulation greater than $80 \mathrm{~mm}$, mean temperature between $18^{\circ} \mathrm{c}$ and $32^{\circ} \mathrm{c}$. The results of this study show that seasonality of climate influences the variation in malaria transmission in the district. It was observed that rainfall played an important role thereby influence malaria transmission. Monthly precipitation greater than $80 \mathrm{~mm}$ was associated with increased malaria transmission rates in the district, and temperature probably was not a limiting factor.

There was no district-based epidemic early warning, prevention and response, reported by the district.

Malaria prevalence and climatic factors showed significant relation.By monitoring malaria cases and supplementing this information; it is possible to make educated and informed judgments on the state of the malaria season and possible outbreaks.

A prediction based on seasonal climate forecasts that are available in advance of the main transmission season is important to prevent the impact of malaria. These forecasts can predict overall likelihood of whether a particular rainy season will be above or below average.

A strong early warning system can allow last minute preparedness measures and early response to outbreaks, saving lives and reducing pressure on the health system in the district. Climate data monitoring should be put the spot light, which should respond by increased disease surveillance (weekly reporting for instance) in order to pick up a possible outbreak at the earliest stages. Public health emergency management at different scales of the health system could raise warning flags signaling the need for increased level of alertness, preparedness and proactive interventions. We recommend the development of climate prediction models to help forecast and control malaria outbreaks in the district

\section{References}

[1] World Health Organization. Malaria epidemic assessment and reporting: Field guide for field testing 2004.

[2] Jacob M. PN, Abdulmumini U., et al. Roll back malaria. African success story in Eritrea. January 2007; 97.

[3] Pampana E. A text book of malaria eradication: Oxford University Press; 1969.

[4] Malaria epidemics: Forecasting, Prevention, Early detection and Control 
[5] Ethiopian Ministry of Health in collaboration with World Health Organization. Guidelines for the prevention and control of selected epidemic diseases in Ethiopia August 2002 .

[6] Climate variability and malaria transmission: A case of Nigeria.

[7] Ethiopian Ministry of Health. Malaria indicator survey 2007. 2007.
[8] Ethiopian Ministry of Health. National malaria guideline. August 2011.

[9] Hailay D., Joel S., Awash T., and Marc L. Alert threshold algorithms and malaria epidemic detection.

[10] Systems for the early detection of malaria epidemics in Africa. 